(Aus dem physiologischen Institute in Bonn)

\title{
Die quantitative Bestimmung von Fetten, Seifen und Fettsäuren in thierischen Organen ${ }^{1}$ ).
}

Von

Dr. phil. C. Dormeyer.

Es scheint eine allgemein anerkannte Annahme zu sein, dass zur Bestimmung der Fette in thierischen Organen die Extraction mittelst Aether eine zuverlässige und genaue Methode ist. Die Angaben in der Litteratur lauten dahin, dass man zur Bestimmung des Fettgehaltes die getrockneten Organe mit heissem Aether extrahiren soll. Während nun schon die Angaben über die verschieden lange Zeitdauer der Extraction es vermuthen lassen, dass unter sonst gleichen Bedingungen demgemäss auch die Menge der Extracte veränderlich ist, so findet man doch bis in die neuere Zeit jene Methode zur Bestimmung der Fette angewandt. Auf die Frage nun, worauf sich denn die Genauigkeit dieser Methode grïnde, welche Kriterien man habe, dass das Fett mittelst siedenden Aethers aus den Organen vollständig herausgeholt werden kann, darauf sucht man in der Litteratur vergebens nach einer Antwort. Ich brauche nun kaum darauf hinzuweisen, dass es unbedingt nothwendig ist, die Genauigkeit jener Methode in ihrer Grundlage zu prïfen, da die Möglichkeit einer genanen Bestimmung dieses Reservestoffes der Organe von grundsätzlicher Bedeutung ist.

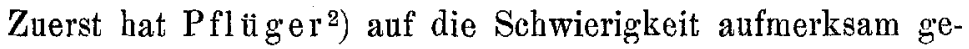
macht, der man begegnet, wenn man getrocknetes Pferdefleisch mit siedendem Aether an Extract erschöpfen will. Er präcisirte seine Meinung uber obige Methode dahin: "Aus allen angefïhrten Gründen halte ich die genane quantitative Bestimmung der Fette

1) In einer vorläufigen Mittheilung (Pf. Arch. 61, 341) babe ich das Wesentliche dieser Arbeit schon bekannt gegeben.

2) Pf. Arch. 51, 277. 
Die quantitative Bestimmung von Fetten etc. in thierischen Organen. 91

mit unseren heutigen Methoden nicht blos für.schwierig, sondern

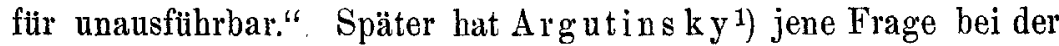
Bestimmung des Fettgehaltes von in vacuo getrocknetem Ochsenfleisch wieder aufgenommen. Er theilt mit, dass er manchmal genöthigt gewesen sei, das getrocknete Fleisch eine Woche lang zu extrahiren und zwar betrug nach ihm der Fettgehalt des Aetherextractes aus 10 bis $20 \mathrm{~g}$ Substanz am fünften und sechsten Tage nur wenige Milligramme. „Auf diesem Punkte angelangt habe ich in der Regel die Extraction als beendet angesehen, da es mir auch nach weiter fortgesetzter Extraction nie gelang, den Aether frei von jeglichem Rückstand zu erhalten." Obwohl nun Argutinsky selbst die Berechtigung des Einwandes, dass die Summe von selbst Milligrammen des Extractes viele Tage beobachtet eine wohl zu berïcksichtigende Menge bilden, für berechtigt erachtet, so meint er doch, "dass eine eine Woche danernde Fettextraction mit schuell circulirendem, warmen Aether im Soxhlet'schen Apparate wohl die Grenze dessen erreicht, was man practisch verlangen kann." Es,wird sich jedoch aus den in der folgenden Aufstellung I mitgetheilten Ergebnissen erweisen, dass selbst Argutinsky noch weit davon entfernt war, den Fettgehalt des Fleisches erschöpft zu haben.

Ich werde nunmehr die in den folgenden drei Aufstellungen I A, B, C mitgetheilten Versuchsergebnisse näher erläutern und einer eingehenden Betrachtung unterziehen.

Verschiedene Muskeln - vergl. Aufstellung I - eines im Stoffwechselgleichgewicht befindlichen $30 \mathrm{~kg}$ schweren Hundes wurden auf das Sorgfältigste, dies betone ich bésonders, von allen makroskopisch sichtbaren Fetttheilchen befreit und dann in vacuo über Schwefelsäure getrocknet. Die Muskeln, welche sich so recht schnell und ohne merkbare Veränderung trocknen lassen, wurden nunmehr nach Möglichkeit zerkleinert. Dies gelingt jedoch, hierin bestätige ich Argutinsky's Angabe, wegen des vorhandenen Fettes immer nur ganz annähernd. Das verhältnissmässig grobe Pulver wurde nunmehr in eine Papierhülse gefüllt und mehrere Stunden mit siedendem Aether im Soxhlet'schen Apparate behandelt. Der Aether circulirte so schnell als zulässig war; er hatte im Apparate eine Temperatur von $30^{\circ} / 32^{\circ}$. Der

1) Pf. Arch. 55, 6. 
I.

A. Behandlung von

$50 \mathrm{~g}$ Musc. gluteus

wäbrend einer Versuchszeit von 863 Stunden mit heissem Aether.

\begin{tabular}{|c|c|c|c|}
\hline $\begin{array}{c}\text { Behandlung } \\
\text { vor der } \\
\text { Extraction }\end{array}$ & 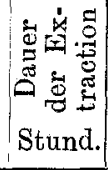 & 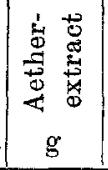 & Durch Verseifung erhalten \\
\hline $\begin{array}{c}\text { Pulverisirt } \\
" \\
" \\
\text { Nicht pulvers. } \\
" \\
\text { Pulverisirt } \\
" \\
" \\
" \\
" \\
\text { 350 St. ohne P1 } \\
\text { sation unter } \\
\text { Nicht pulvers. } \\
\text { Pulverisirt } \\
n \\
\text { Nicht pulvers. } \\
" \\
" \\
" \\
\text { Pulverisirt } \\
"\end{array}$ & \begin{tabular}{|c|}
15 \\
14 \\
10 \\
26 \\
20 \\
31 \\
33 \\
30 \\
42 \\
46 \\
33 \\
45 \\
39 \\
Aulveri- \\
Aether. \\
37 \\
39 \\
39 \\
37 \\
57 \\
46 \\
56 \\
37 \\
37 \\
51 \\
43 \\
\end{tabular} & $\mid \begin{array}{l}3,1500 \\
0,4900 \\
0,2600 \\
0,0900 \\
0,0399 \\
0,0502 \\
0,0395 \\
0,0853 \\
0,0900 \\
0,1116 \\
0,0592 \\
0,0750 \\
0,0600 \\
0,0047 \\
0,0420 \\
0,0729 \\
0,0522 \\
0,0649 \\
0,0300 \\
0,0150 \\
0,0150 \\
0,0085 \\
0,0066 \\
0,0245 \\
0,0170\end{array}$ & $\begin{array}{l}0,0200 \mathrm{~g} \text { Fettsäuren, } 0,0101 \mathrm{~g} \text { Cholesterin. } \\
0,0691 \mathrm{~g} \text { Fettsäuren. } \\
0,0502 \mathrm{~g} \text { Fettsäuren.; davon } 0,0201 \mathrm{~g} \text { als } \\
\text { freie Fettsă }\end{array}$ \\
\hline Summ & 863 & $\overline{9540}$ & \\
\hline
\end{tabular}

zu diesem Zwecke dienende Aether war rein und enthielt keine nachweisbare Menge von Säure. Durch diese erste Extraction erhielt man, wie sich aus den drei Aufstellungen I ergibt, etwa $3 / 5$ der gesammten extrahirten Menge des Fettes. Alsdann wurde nach Entfernung des Aethers die Masse auf's Neue pulverisirt und schon jetzt eine ziemliche Feinheit des Pulvers erreicht. Die Extraction mit Aether wurde alsdann wiederholt. Dieses Verfahren wurde 3-4 Mal vorgenommen und dabei immer noch durchaus bemerkenswerthe Mengen von Aetherextract erhalten. Um den Einwand zu beseitigen, als bätte man durch ein einmaliges, aber weiter- 
Die quantitative Bestimmung von Fetten etc. in thierischen Organen. 93

I.

B. Behandlung von

38 g Mnse. quadriceps (rechts)

während einer Versuchszeit ron 871 Stunden mit heissem Aether.

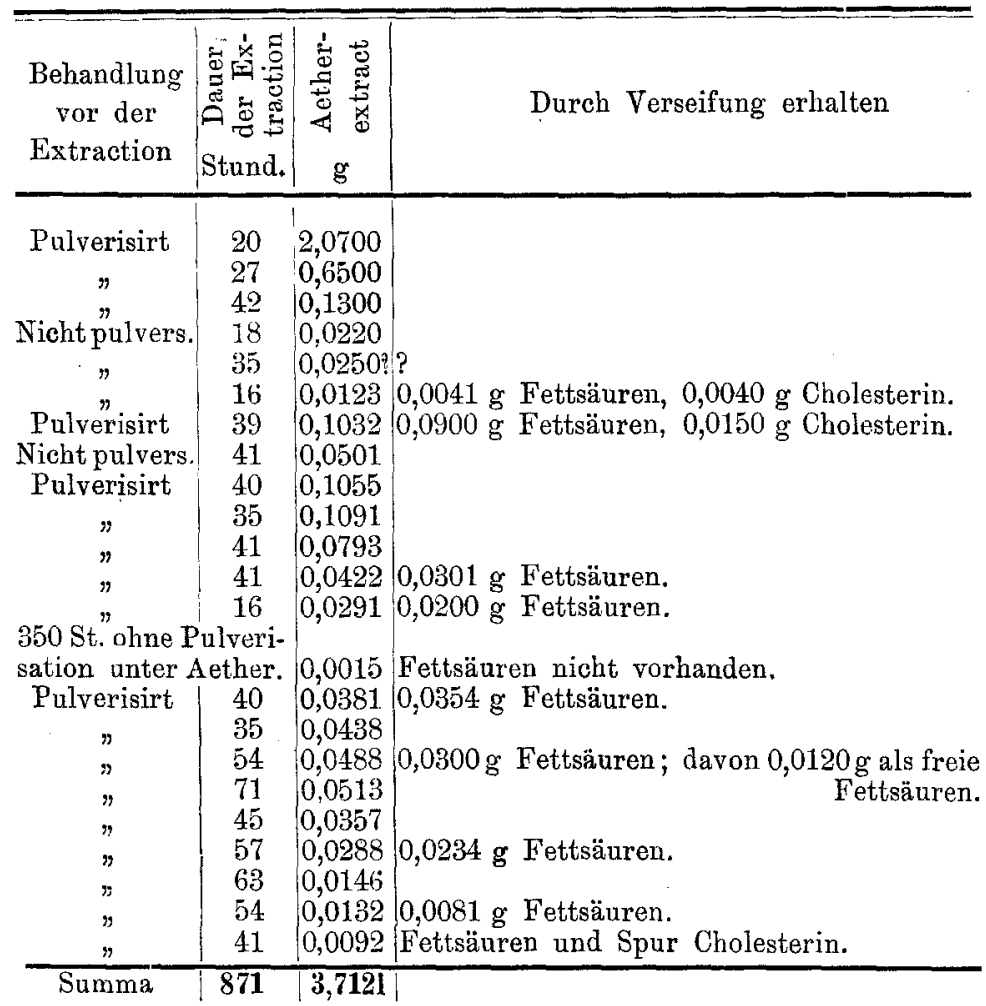

grehenderes Pulverisiren die ganze Menge des in den 3-4 letzten Malen erhaltenen Aetherextractes auf einmal gewinnen können, genügt es darauf hinzuweisen, dass auf das Pulverisiren von je vier Gramm Substanz etwa eine halbe Stunde verwandt wurde. Und weiter, da es nicht gelingt, das Bindegewebe im Mörser hinreichend zu zerkleinern, so wurde dieses mittels einer Scheere zerschnitten. Hierbei wurde sogar, um kleine Theilchen des Bindegewebes aufzufinden, eine Lupe benutzt. Wie die drei Aufstellungen I ergeben, war der Aetherextract immer noch erheblich, aber stets abnehmend. Es wurde nunmebr das Pulverisiren ausgesetzt und das Pulver, welches schon einen bedeutenden Grad von Feinheit erlangt hatte, weiter mit Aether extrahirt. Auch hierbei wurden immer noch nicht zu 
I.

\section{Behandlung von \\ 32 g Musc. quadriceps (links)}

während einer Versuchszeit von 1044 Stunden mit beissem Aether.

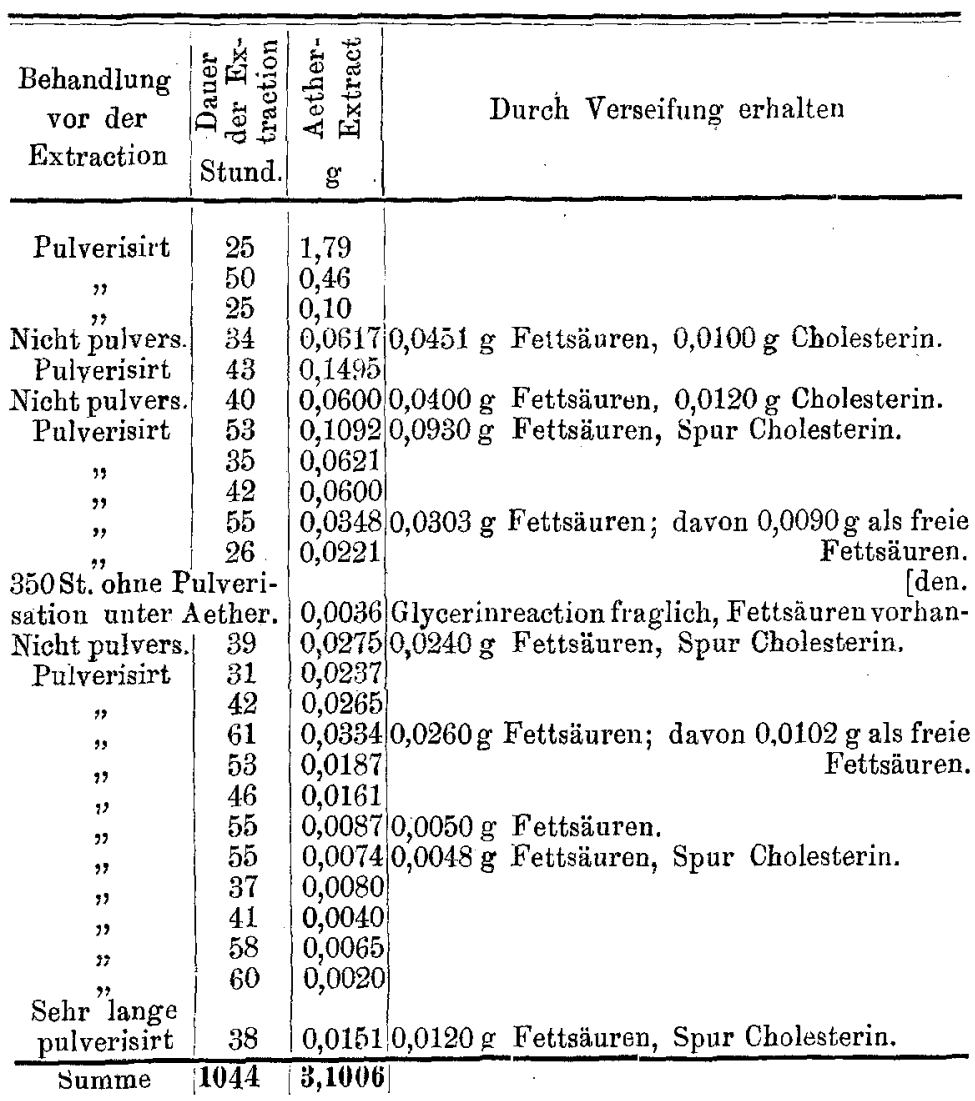

vernachlässigende Mengen Aetherextract gewonnen. Setzte man dies Verfahren, also ohne das Organpulver vorher zu pulverisiren, fort, so nahm der Extract auch langsam ab. Wurde jedoch dasselbe vor der Behandlung mit Aether auf's Neue pulverisirt, so ergab sich die bemerkenswerthe Thatsache, dass durch den Aether 2,5, ja 8,5 mal so viel Extract herausgebolt wurde, als beim vorhergebenden Male, wo das Fleisch nicht pulverisirt worden war. Dieses Verfahren wurde noch mehrmals wiederholt und zwar mit dem verhältnissmässig gleichem Erfolge. Das Fleischpulver hatte schon, bevor die erste 
Hälfte einer Versuchsreihe beendigt war, einen solchen Grad ron Feinheit erreicht, dass bei dem jedesmaligen erneuten Pulverisiren die grössere Fcinbeit des Pulvers nicht ermittelt werden konnte. Es steht jedoch fest, dass nur durch das erneute Pulverisiren eine weitere Menge Extract gewonnen werden konnte und demnach auch die Muskelsubstanz durch das Pulverisiren einen böheren Grad von Feinheit erreicht haben musste. Denn ward nunmehr weiterhin das Pulverisiren ausgesetzt, so sank der Extract sogleich bedentend und stieg dagegen im Verbältniss zu jenem bei erneutem Pulverisiren. Obiges Verfahren wurde fortgesetzt in der Erwartung, dass man schliesslich doch zum Ziel gelangen würde. Trotz der ganz bedeutenden Extractionszeit von 900-1000 Stunden ${ }^{1}$ ) gelang es aber nicht, das Fleisch an Aetherextract zu erschöpfen, denn die zum Schlusse.erhaltenen Mengen sind doch noch so bedeutend, dass sie unbedingt in Betracht kommen müssen. Wenn sich nun auch unter Zugrundelegung der in der folgenden Aufstellung II angegebenen Zahlen ermitteln lassen würde, wie lange Zeit man noch aufwenden müsse, um den zu begehenden Fehler möglichst klein zu machen, so ist dies aus practischen Gründen unausführbar.

II.

In je 10 Stunden aus :

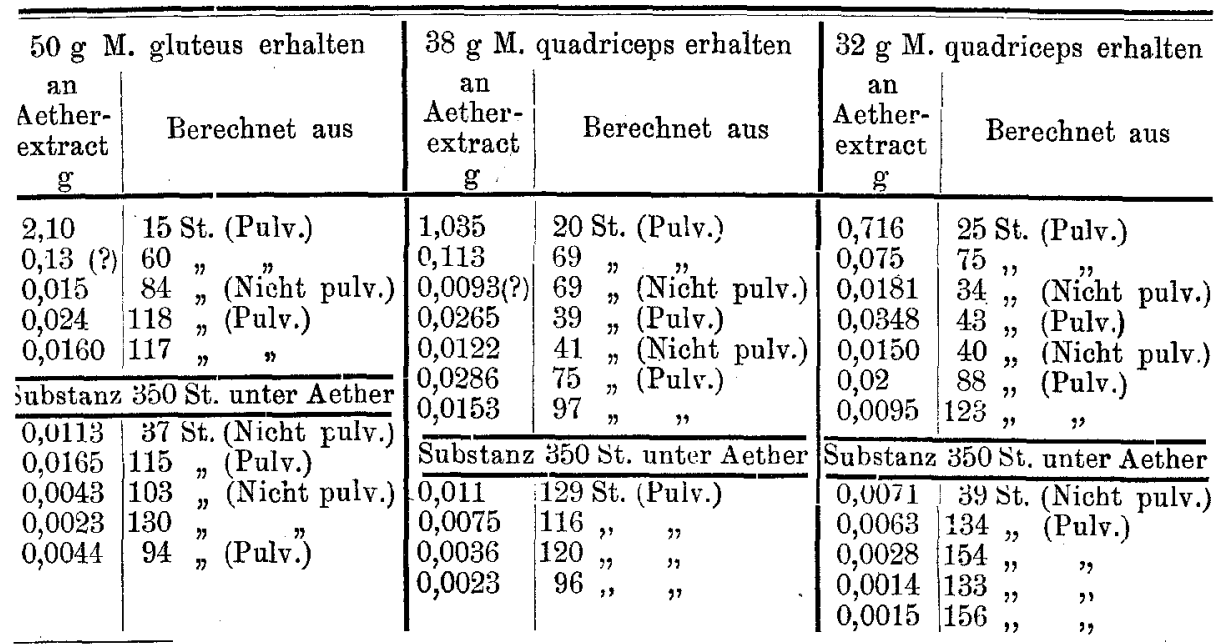

1) Ich will nicht unerwähnt lassen za bemerken, dass diese Anzahl von Stunden einer Versuchsdauer von vielen Monaten entsprechen, da ja wegen der Feuergefährlichkeit des Aethers die Extraction nicht während der Nacht fortgesetzt werden durfte. 
Aus dieser Aufstellung geht deutlich das dauernde Fallen des Aetherextractes hervor.

Der Einwand, dass die so auffällig langwierige Aetherextraction ihre Ursache habe in einer individuellen Eigenthiumlich. keit, in einem ganz besonders grossen Fettreichtum des Versuchsgegenstandes, wird dadurch beseitigt, dass ein zweiter Versuch mit den Muskeln eines anderen Hundes dasselbe Ergebniss hatte. Dieser Versuch wurde jedoch nur so lange fortgesetzt als er von grundsätzlicher Bedentung war. Versuche, mit Ligroïn schneller zum Ziele zu kommen, hatten ein negatives Ergebniss.

Bei der Beurtheilung der Frage, weshalb der Aether nur so äusserst schwierig das Fett aus der trocknen Muskelsubstanz herausholen kann und zwar auch dann nur in begrenzter Menge, steht zunächst fest, dass es darauf ankommt, dem Aether immer neue Oberflächen darzubieten. Dies gesehieht eben durch das Pulverisiren, indem so das Gewebe in seinem Zusammenhange und die einzelnen Zellen selbst zerstört werden. Der Umstand, dass schon vom Aether in Lösung gebrachtes Fett durch das Organpulver selbst zuriickgehalten werden kann, wird auch dazu beitragen, die Erschöpfung an Fett zu erschweren. Die Thatsache ferner, dass es innerhalb weniger Stunden gelingt, etwa $\% / 5$ der gesammten extrahirbaren Menge des Fettes $\mathbf{z} \mathfrak{u}$ erhalten und der dann noch vorhandene Theil nur ganz allmählich gewonnen werden kann, weist darauf hin, dass es verschiedene Arten des Fettvorkommens in den Muskeln gibt. Man kann sich nämlich vorstellen, dass das leichter Extrahirbare denjenigen Theil des Fettes darstellt, der als Reservestoff in dem interstitiellen Bindegewebe vorhanden ist. Diejenige Menge dagegen, welche der Aetherextraction so hartnäckigen Widerstand entgegensetzt und vollständig erst nach gänzlicher Desorganisation des Gewebes gewonuen werden kann, demjenigen Theil entspricht, der sich in dem Muskelparenchym selbst vorfindet.

Es ergibt sich somit, dass man auf dem Wege der Extraction mit Aether keine quantitative Bestimmung des Fettes und der Fettsäuren ausfïhren kann. Die Bestimmung der Seifen würde auf diese Weise überhanpt nicht möglich sein. Um jene auszufübren, bleibt demnach nur die Möglichkeit, die Organe nafzaschliessen". Es gelingt dieses reeht vortheilhaft dureh Pepsin-Salz- 
Die quantitative Bestimmung von Fetten etc, in thierischen Organen. 97

säure $\left.{ }^{1}\right)$. Bei Anwendung dieses weiterhin ausführlich mitgetheilten Verfahrens wurden in den obigen drei Muskeln noch die in der Aufstellung III angegebenen Fettmengen ermittelt.

III.

\begin{tabular}{l|c|c|c|c}
\hline & $\begin{array}{c}\text { Angewandte } \\
\text { Substanz }\end{array}$ & Fett $\left.{ }^{2}\right)$ & $\%$ & $\begin{array}{c}\text { Daraus an } \\
\text { Fettsäuren } \\
\text { durch } \\
\text { Verseifung }\end{array}$ \\
\hline Musc. gluteus & 2,0015 & 0,0182 & 0,91 & 0,0160 \\
Musc. quadriceps (rechts) & 2,3461 & 0,0200 & 0,85 & 0,0170 \\
Musc. quadriceps (links) & 2,2477 & 0,0171 & 0,81 & 0,0150 \\
& & 0,0189 & 0,84 & 0,0152
\end{tabular}

Es ergibtsich demnach diebemerkenswerthe Thatsache, dass aus den obigen drei Monate lang mit Aether behandelten Muskeln vermittelst der peptischen Verdau ung noch etwa 8,5\% der Gesammtmenge der Fette, Seifen und Fettsäuren erhalten wurden. Ist aber in dem untersuchten Organpulver noch eine so bedeu. tende Menge von diesen Körpern enthalten, wie viel mehr wird dann den bisherigen Autoren bei der Bestimmung des Fettes mittelst der Extractionsmethode entgangen sein? Die bisherigen Fettbestimmungen in thierischen 0 rganen sind deshalb weit davon entfernt, einen auch nur an näherungsweise richtigen Wertb für die Menge der Fette und Fettsäuren zu ergeben.

Was nun den Aetherextract betrifft, so ist bekannt, dass ausser dem Fett und den höheren Fettsäuren noch eine Anzahl

1) Die vorliegende Untersuchung war abgeschlossen, als mir eine Arbeit von $\mathrm{R}$ adzi ejewski (Vir ch. Arch. 1868. 43. 268) überFettresorption bekannt wurde. In derselben gibt er kurz an, dass er die künstliche Magenverdauung zur Bestimmung des Fettes im Fettgewebe und im Muskel verwandt habe, ohne dass er irgend eine Mitheilung dariber macht, weshalb man grundsätzlich dies Verfahren anwenden muss. Sein primitives Ver. fahren, das ganz in Vergessenheit gerathen za sein scheint, hat er keineswegs ausgearbeitet. In der vorliegenden Form ist es für quantitative Zwecke nicht brauchbar.

2) Ieh setze hier absichtlich für den Aetherextract die Fette und Fett: sänren ein. Der Beweis hierfür findet sich weiter unten; er ergibt sich weiter aus der Thatsache, dass man durch Verseifung die berechnete Menge an Fettsäuren erhielt. 
anderer Substanzen in grösserer oder geringerer Menge in denselben bei der Behandlung von getrocknetem Fleisch mit siedendem Aether übergehen. Dahin gehören die Lecithine, das Cholesterin und seine Verbindungen und stickstoffhaltige Extractivstoffe.

Ich bemerke zunächst allgemein, dass die erhaltenen Aetherauszüge im Anfang dickflüssig waren in Folge des vorherrschend vorhandenen Oleïns. Nach mehrtägigem Stehen schieden sich aus denselben weisse, körnige Massen aus, die aus Stearin bestanden. Daneben waren freie Fettsäuren vorhanden. Nachdem das Fleischpulver etwa 500 Stunden extrahirt worden war, erhielt man Auszüge von festerer Beschaffenheit. Ich will nicht unterlassen zu bemerken, dass es immerhin längere Zeit dauerte bis die Aetherausziuge ein constantes Gewicht besassen. Die hierbei ermittelte Gewichtsabnahme steigerte sich bei den nach einander erhaltenen Aetherauszügen. Diese Fliichtigkeit einzelner Bestandtheile der Auszüge weist darauf hin, dass man es hier mit niederen Fettsäuren zu thuen hat. Bei den in den drei Aufstellungen I angegebenen Werthen sind nur die aus dem constanten Gewicht ermittelten Zahlen berücksichtigt worden. Beim Verdunsten des Aethers der nach der 100 stdg. Extractionszeit erhaltenen Auszitge auf dem Wasserbade entwickelte sich ein unangenehmer, stechender, die Schleimbäute reizender Körper, der Acroleïn za sein scheint.

Da nun die in den ersten Stunden der Extractionszeit erbaltenen Aetherauszüge schon mehrmals von anderen Seiten untersucht worden sind und es für meine Zwecke schliesslich nicht darauf ankam, wie viel Fett im Ganzen durch die Extraction erbalten worden war, so habe ich mich begnügt, einzelne mir besonders bemerkenswerthe Ausziige auf Fett, Fettsäuren und Cholesterin zu untersuchen. Es ist dadurch der Nachweis des Vorhandenseins von Fett, Fettsäuren und Cholesterin - siebe Aufstellung I - erbracht worden. Mit jedem einzelnen Auszuge ${ }^{1}$ ) wurden jedoch die qualitativen Reactionen auf Glycerin angestellt. Diese Reactionen hatten nur ausnahmsweise ein negatives Ergebniss. Dies trat bemerkenswerther Weise mehrmals dann ein, wenn die Muskelsubstanz vor der Extraction nicht aufs Neue pulverisirt worden war. In solchen Fällen enthielt der Auszug ${ }^{2}$ )

1) Soweit sie nicht verseift wurden.

2) Argutinsky (1. c.) macht schon darauf aufmerksam, „dass der spärliche Rückstand der letzten Tage krystallinischer war". 
Die quantitative Bestimmung von Fetten etc. in thierischen Organen. 99

schon nach dem Verjagen des Aethers deutlich sichtbare, manchmal concentrisch angeordnete lange Nadeln. Die Verseifung ergab das Vorhandensein von Cholesterin und Fettsäuren. Es ist wahrscheinlich, dass das Cholesterin nicht nur als solches vorhanden gewesen sein wird, sondern zum Theil in der Form der von $\mathrm{H} u ̈ \mathrm{rtle}^{1}$ ) im Blut nacbgewiesenen Fettsäureester. Der Gesammtaetherauszug eines zweiten Theiles des Musc. gluteus des untersuchten Hundefleisches enthielt, nach der Methode von $\mathrm{Ober-}$ $\mathrm{m} \mathfrak{i} 1 \mathrm{l}$ e $\mathrm{r}^{2}$ ) behandelt, $0,0277 \mathrm{~g}$ Cholesterin. Da $12 \mathrm{~g}$ trockner Muskelsubstanz angewandt worden waren, so enthielt dieselbe $0,23 \%$ Cholesterin.

Lecithin wurde nur qualitativ durch das Vorhandensein von Phosphor nachgewiesen.

Was den Stickstoffgehalt der Auszüge betrifft, so ergibt sich aus der Aufstellung IV, dass dieser sehr gering ist; mit den nach einander erhaltenen Auszïgen nimmt derselbe zu.

IV.

a) Musc.gluteus.

\begin{tabular}{|c|c|c|c|c|c|c|}
\hline & Aetherextract & 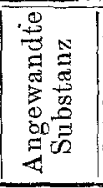 & 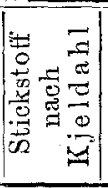 & $\begin{array}{c}\text { Stickstoff } \\
\text { berechnet für } \\
4,2949 \mathrm{~g} \\
\text { Aetherextract }\end{array}$ & $\begin{array}{l}\text { Z } \\
\frac{0}{0}\end{array}$ & 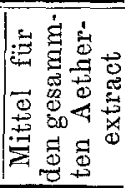 \\
\hline 1) & $\begin{array}{l}\text { Gewonnen durch } 221 \text { stdg. } \\
\text { Behandeln des Muskelpul- } \\
\text { vers mit siedendem Aether. }\end{array}$ & $\begin{array}{l}0,2230 \\
0,2605\end{array}$ & $\begin{array}{l}0,0006 \\
0,0008\end{array} \mid$ & $\begin{array}{l}0,0133 \\
0,0137\end{array}$ & $\begin{array}{l}0,31 \\
0,32\end{array} \mid$ & \multirow[b]{2}{*}{$0,42 \%$} \\
\hline & $\begin{array}{l}\text { Nach weiterer } 642 \text { stdg. } \\
\text { Behandlung. } \\
\\
\text { b. Musc. }\end{array}$ & $\begin{array}{l}0,0750 \\
0,1217\end{array} \mid$ & \begin{tabular}{|}
0,0008 \\
0,0013
\end{tabular} \mid & $\begin{array}{c}\text { Ber. f. } 0,6591 \mathrm{~g} \\
\text { Aetherextract } \\
0,0074 \\
0,0073 \\
\text { s (rechts). }\end{array}$ & $\left|\begin{array}{l}1,12 \\
1,13\end{array}\right|$ & \\
\hline 1) & $\begin{array}{l}\text { Gewonnen durch } 278 \text { stdg. } \\
\text { Behandeln des Muskelpul- } \\
\text { vers mit siedendem Aether. }\end{array}$ & $\begin{array}{l}0,1295 \\
0,1050\end{array} \mid$ & $\begin{array}{l}0,0003 \\
0,0003\end{array}$ & $\begin{array}{c}\text { Ber. f. } 3,1681 \mathrm{~g} \\
\text { Aetherextract } \\
0,0096 \\
0,0104\end{array}$ & $\begin{array}{l}0,31 \\
0,33\end{array} \mid$ & \multirow[b]{2}{*}{$0,43 \%$} \\
\hline 2) & $\begin{array}{l}\text { Nach weiterer } 593 \text { stdg. } \\
\text { Behandlung. }\end{array}$ & $\mid 0,0909$ & $\begin{array}{l}0,0009 \\
0,0010\end{array}$ & $\begin{array}{c}\text { Ber. f. } 0,5440 \mathrm{~g} \\
\text { Aetherextract } \\
0,0058 \\
0,0059\end{array}$ & $\begin{array}{l}1,08 \\
1,09\end{array}$ & \\
\hline
\end{tabular}

1) Z. f. physiol. Chem. 21. 331.

2) Dissertation Berlin 1892. pag. 57. 
Es ergibt sich demnach im Mittel genau derselbe Werth, welchen Petersen ${ }^{1}$ ) im Aetherextract von magerem Rindfieiseh ermittelt hat.

\section{Werden Fette und Seifen durch die peptische Verdaunng gespalten?}

Es handelt sich nun zunächst um die Frage, ob das Fett bei der Einwirkung von künstlischem Magensaft eine Spaltung erleidet. Der durch eine Abspaltung des Glycerins bedingte Verlust ist allerdings so klein, dass man ihn wohl vernachlässigen könnte. Es ist jedoch zu bemerken, dass man dann nicht im Stande sein würde, das Verbältniss der Fette zu den Fettsäuren zu ermitteln. In der letzt erschienenen Arbeit über diese Frage kommt $\mathrm{C}$ o n teje $\mathrm{an}^{2}$ ) im Gegensatz zu den Untersuchungen von $\mathrm{Cash}^{3}$ ), Ogata $a^{4}$ ) und Marpman $n^{5}$ ) zu dem Sehluss, dass „der Magensaft keine verdanende Wirkung auf das Hammelfett ausiubt" 6). Da es für meine Zwecke lediglich darauf ankommt, festzustellen, ob die künstliche Magenverdaung eine Veränderung der Fette bewirkt, so habe ich nur die diesbeziiglichen Versuche von Contejean in Betracht zu ziehen. Er lies zur Ermittelung jener Frage „wochenlang" bei einer Temperatur von $40^{\circ}$ künstlichen Magensaft eines Hundes auf Hammelfett einwirken. Dasselbe ,verlor kein Centigramm an Gewieht". Da nun $\mathrm{Con} t \mathrm{t}$ j e a n nichts dariber mittheilt, wie viel Substanz er in seinen Versuchen angewandt hat, so können jene Centigramm! ebensowohl auf einen grossen wie auf einen ganz geringen Verlust gedeutet werden. Aus diesem Grunde habe ich daher den Versuch der Einwirkung von Pepsin-Salzsäure auf Fett wiederholt, deren Ergebnisse die Aufstellung $V$ enthält.

1) Zeitschr. f. Biologie 7, 175.

2) Arch. de Physiol. 1894. (V.) 6. 127.

3) Du Bois-Reymond's Arch. 1880. pag. 323.

4) Eod. 1. 1881. pag. 515.

5) Münch. med. Wochenschr. 1888. pą. 485.

6) 1. c. pag. 134 . 
Die quantitative Bestimmung von Fetten etc. in thierischen Organen. 101

V.

\begin{tabular}{|c|c|c|c|c|}
\hline $\begin{array}{c}\text { Angewandte } \\
\text { Substanz. }\end{array}$ & $\begin{array}{l}\text { Es werden } \\
\text { hinzugefügt }\end{array}$ & Behandlung & $\begin{array}{c}\text { Wieder- } \\
\text { gewon- } \\
\text { nene Sub- } \\
\text { stanz. }\end{array}$ & $\begin{array}{l}\text { Nachd. Behand- } \\
\text { lung mit } \\
\mathrm{Na}_{2} \mathrm{CO} \mathrm{O}_{3} \text { erhal- } \\
\text { tenes Fett. }\end{array}$ \\
\hline 1) 0,7717 & $\begin{array}{c}0,15 \mathrm{~g} \text { Pepsin und } \\
100 \mathrm{ccm} 0,5 \% \mathrm{HCl}\end{array}$ & $\begin{array}{l}24 \text { Stunden bei } \\
370 / 38^{0}\end{array}$ & 0,7710 & 0,7703 \\
\hline 2) 1,2288 & $"$ & $n$ & 1,2281 & 1,2275 \\
\hline 3) 0,6554 & $\begin{array}{l}0,5 \mathrm{~g} \text { Pepsin und } \\
100 \mathrm{~cm} 0,5 \% \mathrm{HCl}\end{array}$ & $\begin{array}{l}32 \text { Stunden bei } \\
370 / 38^{\circ}\end{array}$ & 0,6542 & 0,6538 \\
\hline 4) 0,8375 & $"$ & $\begin{array}{l}41 \text { Stunden bei } \\
370 / 38^{\circ}\end{array}$ & 0,8370 & 0,8371 \\
\hline
\end{tabular}

Durchaus von Fettsäuren freies Schweinefett wurde mit den in obiger Aufstellung angegebenen Mengen Pepsin(Merk)-Salzsäure $\left.{ }^{1}\right)$ mehrere Stunden bei einer Temperatur von $37-38^{\circ}$ gehalten. Hierbei wurde keine sichtbare Veränderung der verschiedenen Fettproben beobachtet. Durch Behandlung mit Aether wurde dem Gewichte nach die angewandte Menge Substanz wiedergewonnen. Es wurde nunmebr, um die gegebenenfalls vorbandenen Fettsäuren zu bestimmen, eine jede der vier Proben mit einer Lösung von Natriumcarbonat behandelt. Eine Entwicklung von $\mathrm{CO}_{2}$ wurde hierbei nicht beobachtet. War nun schon somit das Nichtvorhandensein von Fettsäuren wahrscheinlich gemacht, so wurdé dieses sicher dadurch festgestellt, dass aus der filtrirten klaren Natriumearbonatlösung auf Zusatz von Schwefelsäure keine Ausscheidung erfolgte. Da nun ausserdem die Gesammtmenge des Fettes auch jetzt in allen vier Versuchen, abgesehen von minimalen, nicht ausschliessbaren Verlusten, wieder gewonnen wurde, so ist damit erwiesen, dass unter den für die Methode der Fettbestimmung in Betracht kommenden Versuchsbedingungen keine Spaltung der Fette eintritt.

Was nun die in den Organen vorhandenen Se ife $n$ anbetrifft, so werden diese, wie ich mich dureh Versuche überzeugt habe, durch die $0,5 \%$ Salzsäure gespalten und demnach mit den schon primär vorhandenen Fettsäuren als solehe bestimmt.

Die Cholesterinester, welche auch za den Fetten zu zählen sind, werden wahrseheinlich nicht gespalten werden.

1) Das angewandte Pepsin war von guter verdauender Kraft. 


\section{Ausführung der Methode.}

Die Organe werden zunächst entweder in vacuo oder bei einer Temperatur von $50^{\circ}-60^{\circ}$ getrocknet, alsdann nach Möglichkeit pulverisirt, gut gemischt und etwa $30 \mathrm{~g}$ derselben mit Aether im Soxhlet'schen Extractionsapparate 4-6 Stunden behandelt. Der hierbei gewonnene Aetherauszug wird, je nach den Anforderungen, die man an die Genauigkeit der Analyse stellt, entweder auf seinen Gehalt an Fetten, Fettsäuren u. s. w. untersucht oder gleich in Anrechnung gebracht. Während man bei manchen Organen auf jene Untersuchung verzichten könnte, darf man sie beim Gehirn und Rüickenmark unbedingt nicht unterlassen. Es ist notbwendig die kurze Aetherextraction der getrockneten Organe der Behandlung derselben mit Pepsin-Salzsäure voraufgehen zu lassen, weil es so möglich ist, in grösseren Mengen Substanz eine Fettbestimmung vornehmen zu können. Somit erhält man auch zuverlässige Ergebnisse, die man nicht erwarten darf, wenn man z. B. im Fleisch den Fettgehalt ermitteln will, da dasselbe, wofern nicht das sichtbare Fett und Bindegewebe abpräparirt worden ist, keine homogene Mischung in Bezug auf Fettvertheilung darstellt. Eine solche Mischung kann man bei den Organen zuverlässig nur dann erreichen, nachdem dieselben die grössere Menge an Fett schon abgegeben haben; dies letzte eben erreicht man schon durch die kurze Extractionszeit. Wie schon früher erwähnt, lässt sich das Organ alsdann erst fein pulverisiren, zum mindesten so fein, dass man eine homogene Mischung, wie sich aus der Uebereinstimmung der weiter unten mitgetheilten Analysen ergibt, als vorhanden annehmen darf. Es ist auch weiterhin aus praktischen Gründen vortheilhaft, die Hauptmenge des Fettes durch Behandlung mit siedendem Aether za gewinnen, da bei Anwendung der directen Verdauung einer Substanz die quantitative Gewinnung grösserer Fettmengen ihre Schwierigkeiten hat, die sich nur bei besonderer Vorsicht und Uebung überwinden lassen. Da ausserdem eine Troekengewichtsbestimmung der Organe gewöhnlich ohnehin gemacht werden muss, so bildet die kurze Extractionszeit keine Erschwerung der Methode.

Ich gehe nun zunächst auf die Gewinnung einer brauchbaren Verdaungsflüssigkeit ein, da von deren Wirksamkeit die Genauigkeit der Analyse naturgemäss abhängig ist. 
Die quantitative Bestimmung von Fetten etc. in thierischen Organen. 103

Zum Zwecke der Aufschliessung der Organe habe ich theils die directen Auszïge der Schweinemägen mit. $0,5 \%$ Chlorwasserstoffsäure verwandt, theils Pepsinpräparate benutat. Was die ersten anlangt, so wurde der friseh einem Schweine entnommene Magen mit Wasser gereinigt. Alsdann wurde die Magenschleimhaut entweder abpräparirt und dann in kleine Stückchen geschnitten oder die oberen Schichten derselben mit einem stumpfen Messer abgekratzt und die so gewonnene Masse zusammen mit etwa $600 \mathrm{ccm} \quad 0,5 \%$ Chlorwasserstoffsäure $1-4$ Stunden einer Temperatur von $37 \% / 38^{\circ}$ ausgesetzt. Die etwas schleimige Flüssigkeit wurde alsdann collirt und weiter noch filtrirt. Die auf dem Collirtuche bleibende Masse kann dann noch einmal zur Herstellung einer brauchbaren Verdauungsflüssigkeit verwandt werden. Die auf diese Weise erhaltene Verdaunngslüssigkeit war von durchgehends ausgezeichneter peptischer Wirkungsfähigkeit und zwar reichten je $100 \mathrm{ccm}$ derselben hin, um die zur Analyse nöthige Menge getrockneten Organes gut und schnell aufzulösen. Da die Verdaungsflüssigkeit nun selbst nicht frei von Fett ist, so muss in ihr in derselben Weise, wie weiter unten für das Organ selbst angegeben ist, dasselbe bestimmt werden. Ich erhielt hierbei aus mehreren Analysen ein und desselben Magenauszuges Werthe, die von einander nur um 0,0010-0,0015 g verschieden waren. Der Auszug bestand aus Fett. Bei Anwendung eines Auszuges aus Hundemägen wurde eine grössere verdauende Kraft dieses gegenüber denen vom Schweine ermittelt. Da man jedoch gewöhnlich solche Ausziuge nicht zur Verfügung hat, so erscheint nuir die Benutzung der Auszüge des Schweinemagens am wohlfeilsten und recht zweckentsprechend. Es ist nun nicht von der Hand zu weisen, dass die jedesmalige Herstellung der Auszüge der Magenschleimbaut und die Bestimmung des primär in ihnen vorhandenen Fettes umständlich ist. Eine bedeutende Zeitersparniss gewinnt man bei der Benutzung von Pepsinpräparaten. Es wurden dieselben angewandt in $0,1 \%$ Lösung ${ }^{1}$ ) in $0,5 \%$ Chlorwasserstoffsäure. Allerdings muss ich bemerken, dass manche Pepsinpräparate des Handels viel zu wünschen übrig lassen, sodass man ein Präparat auf seine verdanende Kraft jedesmal untersuchen muss. Diese Prüfung muss von Zeit zu Zeit mit demselben

1) Conf. K 1 u g, Pflüg. Arch. 60. 43 . 
Präparate wiederholt werden. Hat man ein gut verdauendes Pepsinpräparat zur Verfügung, so ist die Benutzung desselben allem anderen schon aus dem Grunde vorzuziehen, weil die Bestimmung des primären Fettes nur 1 bis 2 Mal ausgeführt zu werden braueht und; wenn solches überhaupt vorlanden ist, durch die gewöhnlich ganz minimale Menge desselben die Methode an Schärfe gewinnt.

Ich kehre nunmehr zur Methode selbst zuruick. Da es in Folge nicht hinreichenden Trocknens der Organe vorkommen kann, dass die Differenz im Gewicht zwischen der ursprünglich ermittelten Trockensubstanz und der nunmehr extrahirten grösser ist als das Gewicht des Aetherauszuges, so muss das Gewicht des extrahirten Organpulvers in solch einem Falle auf's Neue ermittelt werden, um einen aliquoten Theil desselben zur Verdaung benutzen zu können. Zur Erzielung einer möglichst gleichmässigen Mischung und zur Unterstützung der Verdauungsflüssigkeit pulverisirt man nunmehr noch das extrahirte Pulver, fügt $\mathrm{zu} 2-4 \mathrm{~g}$ desselben $100 \mathrm{ccm}$ der oben angegebenen Verdaungsflüssigkeit und setzt das Ganze einer Temperatur von $37 \% / 38^{\circ}$ aus. Bei Anwendung eines guten Magenschleimhantanszuges ist das Pulver oft schon in $3 / 4-2$ Stunden verdant und kann weiter verarbeitet werden. Bei Anwendung eines Pepsinpräparates muss man die Einwirkungszeit gewöhnlich bedeutend verlängern, auf zehn, selbst bis auf 24 Stunden. Bei einiger Uebung kann man sich ohne Mühe ein Urtheil uiber den Grad der Verdauung bilden; durch einen etwaig vorhandenen voluminösen, feinen, schleimigen Niéderschlag, der sich oft in Folge der Verdanung bildet, darf, man sich hierbei nicht täuschen lassen. Durch erneuten Zusatz von $0,5 \%$ Salzsäure kann man manchmal eine gestörte Verdaung befördern. Die erhaltene Fliussigkeit kann nun nicht unmittelbar mit Aether ausgeschüttelt werden, sie muss zunächst zur Entfernung des nicht Verdauten - ein geringer Theil wird, wie bekannt, überhaupt nicht verdaut - filtrirt werden. Es gelingt dies recht vortbeilhaft mittelst eines Faltenfilters, auf dessen Boden man einen kleinen Bausch von Glaswolle legt. Um die Filtration zu beschleunigen, ist es zweckmässig, die Fluissigkeit von dem nicht Verdauten abzugiessen und mit warmem Wasser verdünnt zu filtriren. Der eine Theil des Fettes findet sich nunmehr mit dem Rückstande auf dem Filter, der andere in der filtrirten Flïssigkeit. Um den 
Die quantitative Bestimmung von Fetten etc. in thierischen Organen. 105

einen Antheil zu gewinnen, wird das Filter sammt dem Rückstande entweder im Exsiccator oder bei $35^{0} / 45^{\circ}$ getrocknet und dann mittelst heissen Aethers im Soxblet'schen Extractionsapparate erschöpft. Dies kann verschieden lange Zeit, 15-40 Stunden, in Anspruch nehmen. War das Organpulver nicbt gut verdaut und zwar vornehmlich das Bindegewebe zurückgeblieben, so muss man sich durch einen erneuten Verdaungsversuch iuberzeugen, ob der Filterrïckstand nach der Aetherextraction noch Fett ergibt. Was die Gewinnung des Fettes aus dem obigen Filtrate betrifft, so gelingt dies durch Ausschütteln mit Aether. Es genügt zu diesem Zwecke je 100-150 ecm Aether zu verwenden und das Ausschütteln 4 bis 6 mal zu wiederholen; natürlich so lange, bis der Aether nichts mehr aufnimmt. Die Genauigkeit und Schnelligkeit des Ausschïttelns hängt wesentlich daron ab, dass es gelingt, die aetherische und wässerige Schicht scharf zu trennen. Es gelingt nun durchaus, dies soweit zu erreichen, dass man eine quantitative Bestimmung des Fettes in der Flüssigkeit darauf gründen kann. Bei unvorsichtigem Arbeiten kommt es vor, dass die Grenzschicht von Aether und Wasser durch emulsionsartige Bildungen nicht zu ermitteln ist. Dies lässt sich sicher dadurch vermeiden, dass man zunächst eine kleinere Menge Aether, als oben angegeben ist, zu der auszuschüttelnden Flüssigkeit hinzufügt und einige Male langsam und vorsichtig schiittelt. Zeigt sich auch nur schwache Neigung zur Emulsionsbildung, so unterbricht man unmittelbar das Schütteln, fügt vorsichtig erneut Aether hinzu und lässt nunmehr stehen wis die Schichten sich wieder scharf getrennt haben. Man schïttelt alsdann vorsichtig von Nenem und hat nunmehr gewöbnlich schon erreicht, dass, indem man stufenweise stärker schïttelt, keine Bildung von „Schmieren" mehr auftritt. Treten jedoch nach einmal erneutem Aetherzusatz beim Schütteln wiederum emulsionsartige Bildungen anf, so unterbricht man sofort das Schïtteln und verfährt, wie vorher angegeben worden ist. Auf diese Weise gelingt es nun durchaus, die Ausschuittelung quantitativ auszufübren. Die jedesmalig erhaltene aetherische Schicht, die beim ersten Male immer rosa gefärbt ist, lässt man zunächst durch ein, auch zwei Faltenfilter gehen, um das beigemengte Wasser zu entfernen. Ein Theil des dann noch vorhandenen Wassers setzt sich schnell in Tröpfehen ab, wenn man die aetherischen Auszïge mehrere Minuten in einem Becherglase stehen 
lässt. Der nach dem Verjagen des Aethers der gesammten Aetherauszüge erhaltene Rückstand schliesst nun noch anorganische Bestandtheile ein. Man entfernt zunächst immer noch vorhandenes Wasser und nimmt alsdann den Riickstand mit Aether auf und verjagt den letzteren. Dieses Verfahren muss gegebenenfalls noch einmal wiederbolt werden.

Der so gewonnene Rückstand, vereinigt mit der Menge, die durch Extrahiren des Filters erhalten worden ist, stellt demgemäss die noch durch Verdauung gewonnene Fettmenge des Organpulvers dar. Der Rückstand wird nunmehr endguiltig entweder bei $40^{\circ}$ oder im Exsiccator getrocknet. Soweit es die Muskelsubstanz betrifft, habe ich von anderen Substanzen in jenem Riickstande nur geringe Mengen eines braunrothen Farbstoffes gefunden. Nach der Verseifung ergaben sich für die Fettsäuren fast die theoretischen Werthe, wenn man der Berechnung Oleo-Stearo.Palmitin als vorhanden zu Grunde legt. Eine Untersuchung über die Natur der so erhaltenen Fette scheitert an der geringen Menge derselben.

Zum Beweis für die Zuverlässigkeit der beschriebenen quantitativen Methode gebe ich in der Aufstellung VI einige Analysen von Muskeln dreier Hunde. Die Analysen unter A wurden unter Anwendung des Auszuges der Magenschleimhaut eines Schweines, diejenigen unter $B$ bei Benutzung eines Pepsinpräparates ausgeführt. Um die Uebersichtlichkeit der Aufstellung VI nicht zu beeinträchtigen, ist bei den Angaben der durch Verdauung erhaltenen Fettmenge das primär in der Verdauungsflüssigkeit enthaltene Fett - 1) 0,0050 g, 2) 0,0080 g - schon abgezogen worden. Dies ist jedoch nur der Fall soweit es A betrifft. Bei den diesbeziig. lichen Angaben für B war kein solcher Abzug zu machen, da die benutzte Menge von 0,100 g Pepsin nur 0,001 g Extract enthielt.

Die Uebereinstimmung der Ergebnisse obiger Analysen unter einander verbïrgt die Zuverlässigkeit und Genauigkeit der Methode. Ihre Genauigkeit reicht so weit, dass sie den Anforderungen, die man an die quantitative Bestimmung eines Körpers stellen darf, Genüge leistet.

Die Anwendung der Methode ergibt demnach die Gesammtmenge der in dem untersuchten Organe enthaltenen Fette, Seifen und Fettsäuren. 
Die quantitative Bestimmung von Fetten etc. in thierischen Organen. 107
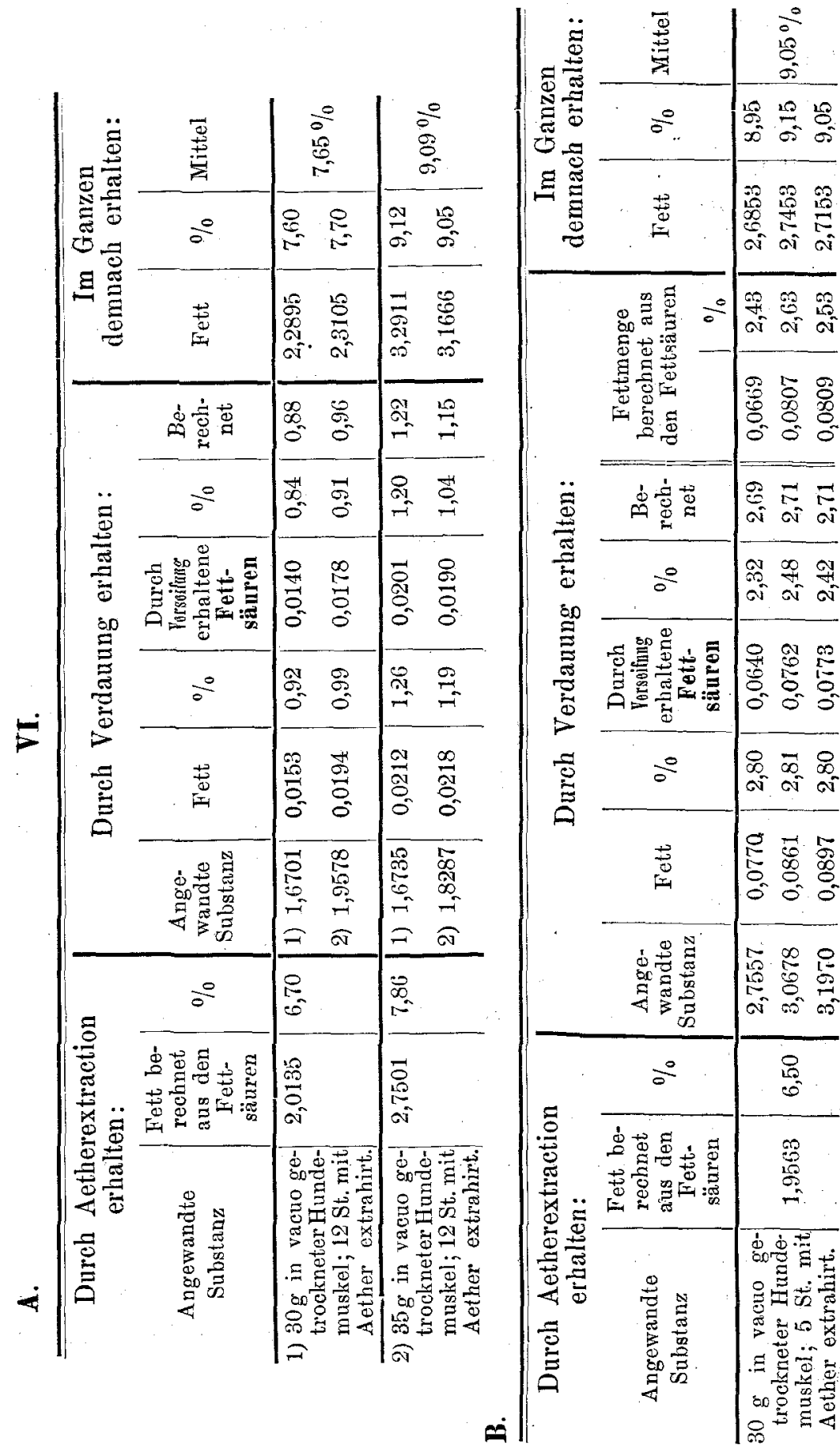
Die Ergebnisse vorstehender Untersuchung fasse ich dahin zusammen.

1. Selbst durch monatelange, tägliche Behandlung der getrockneten und nach grösster Möglichkeit immer auf's Neue pulverisirten Muskelsubstanz mit siedendem Aether gelingt es nicht, dieselbe an Aetherextract zu erschöpfen.

2. In den ersten Stunden des Extrahirens gewinnt man den grösseren Theil des Aetherextractes. Der Rest lüsst sich nur äusserst schwierig und auch nur theilweise aus dem Gewebe herausholen.

3. Der nach monatelangem Extrahiren erhaltene Auszug (Hundemuskel) besteht aus den Glyceriden der Oelsäure, der Stearinsäure und diesen Säuren selbst. Er entbält ausserdem Cholesterin und niedrige, flüchtige Fettsäuren. Lecithin und stickstoffhaltige Extractivstoffe sind nur in geringer Menge vorhanden.

4. Durch die künstliche, peptische Verdauung gelingt es festzustellen, dass in dem monatelang extrahirten Muskel noch etwa $8,5 \%$ der Gesammtmenge der Fette, Seifen und Fettsäuren enthalten sind.

5. Die Glyceride der Fette werden bei der künstlichen, peptischen Verdauung unter den in Betracht kommenden Bedingungen nicht gespalten. Die Seifen werden dagegen zerlegt.

6. Die bisherige Methode zur Bestimmung des Fettes in thierischen Organen mittelst siedenden Aethers ist ganz unbrauchbar, da sie zu niedrige Werthe ergibt.

7. An ibre Stelle tritt die oben beschriebene Methode, welche die Behandlung der Organe mit siedendem Aether mit der nachherigen Anwendung der kïnstlichen, peptischen Verdauung verbindet.

8. Die Controllanalyseil beweisen die Zuverlässigkeit und Genauigkeit der neuen Methode. Dieselbe ergibt die Gesammtmenge der in den Organen enthaltenen Fette (Cholesterin), Seifen und Fettsäuren.

Ich verfehle nicht, auch an diesem Orte Herrn Prof. Dr. E. Pflügjer, der mich zur Bearbeitung dieses Themas aufgefordert hat, meinen Dank für, seine Anregung und gewährte Unterstützung auszusprechen. 